\title{
Silicone Hydrogel Miniscleral Contact Lenses after Corneal Collagen Crosslinking for Post-LASIK Keratoectasia
}

\author{
Boris Severinsky
}

\section{ABSTRACT}

Purpose: To report successful visual rehabilitation of post refractive surgery keratoectasia shortly after corneal collage crosslinking $(\mathrm{CXL})$ procedure achieved with silicone hydrogel mini-scleral (SHmS) contact lens.

Materials and methods: A 29-year-old woman attended to our clinic with complaints on decreased vision in her left eye. Twelve years earlier she underwent bilateral laser-assisted in situ keratomileusis (LASIK) procedure for myopia correction. Corneal topography revealed bilateral central keratoectasia with maximal keratometry values of 55.4 Diopter (D) for her right eye and 59.7 D for the left, corneal thicknesses were 422 and 443 respectively. The patient underwent an uneventful CXL procedure in her left eye and was fitted with SHmS lens 5 weeks later. The lens was designed to rest over the patients' sclera and perilimbal cornea and vault the central cornea with minimal support over it.

Results: SHmS lens fitting resulted in significant subjective improvement in visual acuity (from 20/200, unaided to $20 / 25$, contact lens corrected). The patient was able to wear the lens upto 10 hours a day with stable contact lens corrected vision. No contact lens related complications, such as edema or neovascularization were observed during 3 months follow-up period. As a result of successful restoration of vision in the CXL-treated eye, the patient was scheduled for the procedure in her other eye.

Conclusion: SHmS contact lens should be considered as an option for the visual rehabilitation of corneas shortly after collagen corneal crosslinking procedure. This novel contact lens modality made from flexible and highly gas permeable material minimizes contact lens influence on corneal recovery after CXL and provides an excellent visual outcome.

Keywords: Keratoconus, Corneal collagen crosslinking, Custom soft contact lenses.

How to cite this article: Severinsky B. Silicone Hydrogel Miniscleral Contact Lenses after Corneal Collagen Crosslinking for Post-LASIK Keratoectasia. Int J Kerat Ect Cor Dis 2014;3(3): 127-129.

Source of support: Nil

Conflict of interest: None

Advanced Standing International Program, New England College of Optometry, Boston, Massachusetts, USA

Corresponding Author: Boris Severinsky, Advanced Standing International Program, New England College of Optometry Boston, Massachusetts, USA, e-mail: severinskiy@yahoo.com

\section{INTRODUCTION}

The first paper on corneal collagen crosslinking (CXL) was published by Seiler and Wollensak in 2003. ${ }^{1}$ It is the only treatment that aims to stop or slow down progression of keratoconus $(\mathrm{KC})$ and secondary keratectasia after laser in situ keratomileusis (LASIK) and photorefractive keratectomy (PRK). ${ }^{1,2} \mathrm{Up}$ to date, $\mathrm{CXL}$ is also considered the main vision saving procedure, especially when performed on a young population, when the goal is to prevent possible future keratoplasty.

Studies suggest that CXL has beneficial visual and optical effects by decreasing corneal steepness, improving uncorrected visual acuity, best corrected visual acuity (BCVA) and improving topography irregularity indices. ${ }^{3,4}$ However, a variety of optical devices, such as glasses and special contact lenses (CL), are still necessary to achieve the best corrected vision after CXL.

In the recent years, specialty soft contact lenses for irregular cornea gain increased popularity among eye care practitioners. ${ }^{5}$ One of the bold examples of such lenses is a subtype of well-known Soft-K design, the silicone hydrogel miniscleral lens (Soflex Ltd, Israel). Described lens is a large, $17.00 \mathrm{~mm}$ in diameter, contact lens with center thickness of $0.6 \mathrm{~mm}$. These lenses lathed from Definitive ${ }^{\circledR}$ 74 material (Contamac Corp., UK) with oxygen permeability (DK) of $60 \times 10^{-11} \mathrm{~cm}^{2} \cdot \mathrm{mlO}_{2} / \mathrm{sec} \cdot \mathrm{ml} \cdot \mathrm{mm} \mathrm{Hg}$ (International Organization for Standardization (ISO/Fatt) units and modulus of elasticity of $0.39(\mathrm{~Pa})$. The lenses designed to rest over the patient's sclera, vaults the limbus and the central cornea, having only minimal support on the corneal apex. One of the major uses of silicone hydrogel mini-scleral lenses $(\mathrm{SHmS})$ is visual rehabilitation shortly after CXL. ${ }^{6}$ The goal of such design is to minimize contact lens interference with recovering and remodeling ocular surface, especially the epithelium. The other implication of $\mathrm{SHmS}$ is immediately after refractive or ocular surface reconstruction surgery as a therapeutic, bandage lenses.

\section{CASE REPORT}

Twenty-nine years old women, with the diagnosis of keratoconus, was referred for specially contact lens fitting, due to decreased vision in her left eye for the last 
9 months. Twelve years earlier, she underwent refractive surgery (LASIK) for moderate myopia.

Unaided visual acuities were OD 20/80 and OS 20/200 (Snellen fracture). Patient's refraction was OD +1.25/-3.75 $\times 140$ and OS -5.00/-5.75 $\times 170$ with BCVA of 20/30 and $20 / 80$ respectively. Corneal topography reveals advanced keratoectasia OS > OD, with Maximal Keratometry $\left(\mathrm{K}_{\max }\right)$ of 55.4 diopter (D) in her right eye and $59.7 \mathrm{D}$ in the left (Fig. 1). Central corneal thickness measurements were 422 and 443 , respectively. The patient was also evaluated by cornea specialist and was schedules for a corneal collagen crosslinking, in order to halt the progression of keratoectasia. Temporary spectacles with partial astigmatic correction in the right and 'plano' lens in the left eye were prescribed in order to assist the patient during the postoperative period. Two weeks later, the patient underwent uneventful CXL procedure in her left eye.

Five weeks later, after complete epithelial healing and steroids discontinuation, patient's left cornea exhibits no signs of epithelial hypertrophy or superficial punctate staining and the patient was scheduled for CL fitting. We use a front toric, prism ballasted version of the SHmS lens with a base curve of $7.30 \mathrm{~mm}$ and the power of $-3.75 /-2.25 \times 180$.

On dispensing visit, the BCVA was 20/30, with minor complaint of ghost images. The patient was instructed to build up the wearing hours gradually and was scheduled for a follow-up in the next 10 days. Despite of slight reduction of her visual acuity upon examination (20/50), the patient reported an improvement in overall vision and performance of daily tasks. Patient also reported good lens tolerance and wearing time of 9 hours a day, approximately. Examination reveals a well centered lens with an about $1.00 \mathrm{~mm}$, on-blink movement (Fig. 2). An overrefraction of Plano/-1.50 × 175 was able to improve her vision to 20/25 level and significantly reduce the amount

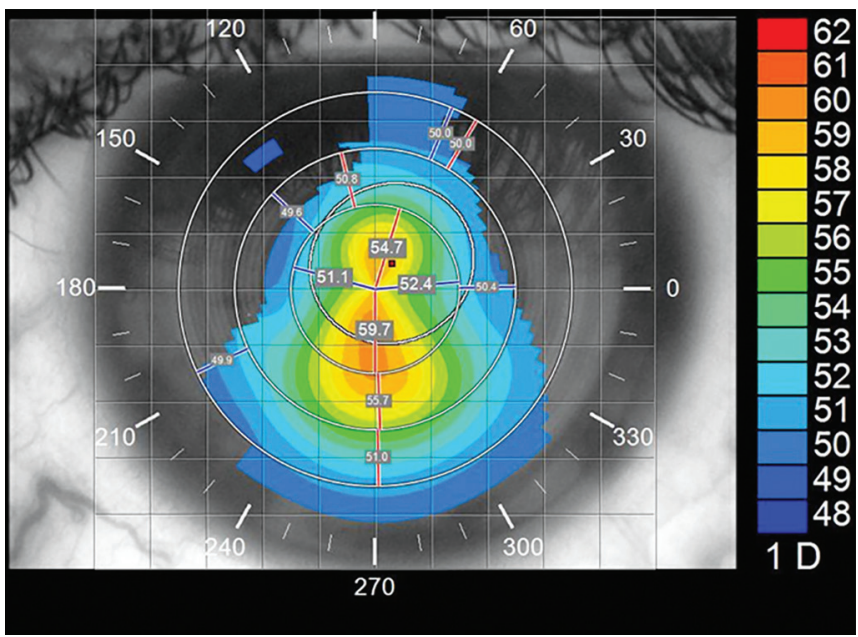

Fig. 1: Corneal topography of the left eye reveals advanced keratoectasia of ghost images. A new lens with an updated power was ordered.

Three months later, the patient was able to wear the lens upto 10 hours a day with stable contact lens corrected vision. The lens covered the cornea completely, centered and moved well. On slit lamp examination, both corneas were clear of signs of punctate staining and central keratometry values remained the same as on the last visit. As a result of successful restoration of vision in the treated eye, the patient was scheduled for CXL in her other eye.

\section{DISCUSSION}

It is known that post-CXL corneal recovery fluctuates over time. Crosslinking studies with long-term follow-up have shown that corneal thickness as well as central keratometry follows a dynamic curve over the first 6 months. ${ }^{7,8}$ Although complete epithelial healing is achieved in the first few days postoperatively, epithelium remodeling is an acceptable explanation for the continuous changes seen over several months. Avoidance of contact lens corneal bearing and mechanical trauma to the center of the cornea seems to be crucial for undisrupted epithelium remodeling and normal corneal nerve proliferation after CXL. Sehra ${ }^{9}$ et al reported that patients fitted with RGP contact lenses 3 months after the procedure shoved evidences of epithelial cell stress with increase in the superficial epithelial cell size and decrease in basal epithelial cell density. These findings were also accompanied by decrease in corneal sub-basal nerve plexus density. The other area of concern is contact lens induced mechanical corneal irritation that may lead to inflammation and consecutive keratocytes apoptosis in the anterior stroma. 10,11

Scleral contact lenses features rooted in SHmS design, provides minimal contact lens interference with the corneal surface. Minimization of corneal contact in turn ensures

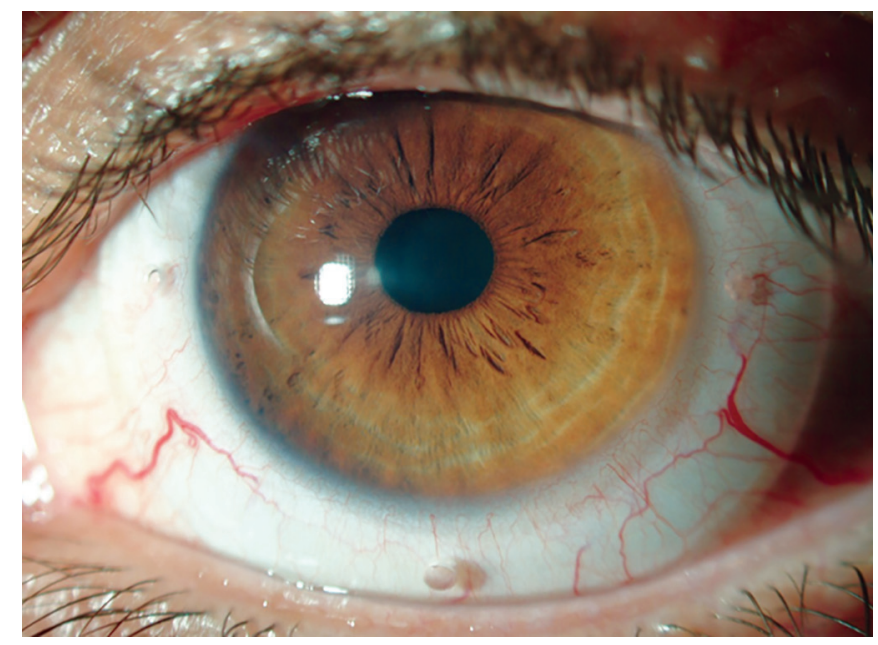

Fig. 2: Proper allocation of the fenestration holes (6 OC) indicates absence of lens rotation 
undisrupted epithelial remodeling, prevents erosions, inflammation, eye rubbing and the risk of infections.

\section{CONCLUSION}

Specialty soft contact lenses possess a clinical importance in today's specialty contact lens practice. The use of this new contact lens design may become a preferred alternative shortly after CXL, since besides visual rehabilitation the avoidance of contact with the central cornea would minimize contact lens influence on epithelial remodeling and allow normal recovery after the procedure.

\section{REFERENCES}

1. Wollensak G, Spoerl E, Seiler T. Riboflavin/ultraviolet- a induced collagen crosslinking for the treatment of keratoconus. Am J Ophthalmol 2003;135(5):620-627.

2. Hafezi F, Kanellopoulos J, Wiltfang R, Seiler T. Corneal collagen crosslinking with riboflavin and ultraviolet A to treat induced keratectasia after laser in situ keratomileusis. J Cataract Refract Surg 2007;33(12):2035-2040.

3. Caporossi A, Mazzotta C, Baiocchi S, Caporossi T. Long-term results of riboflavin ultraviolet A corneal collagen crosslinking for keratoconus in Italy: the Siena eye cross study. Am J Ophthalmol 2010;149(4):585-593.
4. Greenstein SA, Fry KL, Hersh PS. Corneal topography indices after corneal collagen crosslinking for keratoconus and corneal ectasia: One-year result. J Cataract Refract Surg 2011;37(7):1282-1290.

5. Fernandez-Velazquez FJ. Kerasoft IC compared to Rose-K in the management of corneal ectasias. Cont Lens Anterior Eye 2012;35(4):175-179.

6. Severinsky B, Wajnsztajn D, Frucht-Pery J. Silicone hydrogel mini-scleral contact lenses in early stage after corneal collagen cross-linking for keratoconus: a retrospective case series. Clin Exp Optom 2013;96(6):542-546.

7. Greenstein SA, Shah VP, Fry KL, Hersh PS. Corneal thickness changes after corneal collagen crosslinking for keratoconus and corneal ectasia: One-year result. J Cataract Refract Surg 2011;37(4):691-700.

8. Greenstein SA, Fry KL, Bhatt J, Hersh PS. Natural history of corneal haze after collagen crosslinking for keratoconus and corneal ectasia: Scheimpflug and biomicroscopic analysis. J Cataract Refract Surg 2010;36(12):2105-2114.

9. Sehra SV, Titiyal JS, Sharma N, Tandon R, Sinha R. Change in corneal microstructure with rigid gas permeable contact lens use following collagen cross-linking: an in vivo confocal microscopy study. Br J Ophthalmol 2014;98(4):442-447.

10. Kallinikos P, Efron N. On the etiology of keratocyte loss during contact lens wear. Invest Ophthalmol Vis Sci 2004; 45(9):3011-3020.

11. Efron N. Contact lens-induced changes in the anterior eye as observed in vivo with the confocal microscope. Prog Retin Eye Res 2007;26(4):398-436. 